\title{
La « couturière » à la Réunion : figure ou actrice sociale?
}

\section{Reine-Claude GRONDIN}

\section{(2) OpenEdition \\ 1 Journals}

Édition électronique

URL : https://journals.openedition.org/clio/6683

DOI : $10.4000 /$ clio.6683

ISSN : 1777-5299

Éditeur

Belin

Édition imprimée

Date de publication : 1 novembre 2007

Pagination : 209-226

ISBN : 978-2-85816-940-5

ISSN : 1252-7017

Référence électronique

Reine-Claude GRONDIN, « La « couturière » à la Réunion : figure ou actrice sociale? », Clio. Femmes, Genre, Histoire [En ligne], 26 | 2007, mis en ligne le 01 janvier 2010, consulté le 24 avril 2022. URL: http://journals.openedition.org/clio/6683 ; DOl : https://doi.org/10.4000/clio.6683

Ce document a été généré automatiquement le 24 avril 2022

Tous droits réservés 


\title{
La « couturière » à la Réunion : figure ou actrice sociale?
}

\author{
Reine-Claude GRONDIN
}

1 Cet article doit beaucoup à la lecture de l'ouvrage d'Yvonne Verdier Façons de dire, Façons de Faire ${ }^{1}$ et aux remarques de femmes réunionnaises sur le rôle qu'ont pu jouer les mères ou grand-mères couturières dans l'ascension sociale de certaines d'entre elles. Le dépouillement des recensements individuels que nous avions entrepris dans le cadre d'un travail universitaire a confirmé la pertinence du sujet. En effet, l'historiographie de la Réunion ${ }^{2}$ s'est intéressée à la question servile, qui, effectivement, ne peut être ignorée, mais s'est insuffisamment penchée sur la question du travail, hypothéquée par le problème de l'esclavage, et encore moins sur le travail de la femme. L'image de la femme réunionnaise au XIXe siècle est volontiers confondue avec le stéréotype de la «Belle Créole " ${ }^{3}$, «paresseuse jusqu'à l'idéal » comme l'écrit Leconte de Lisle dans sa nouvelle Sacatove, stéréotype qui rassemble tous les traits de la féminité telle qu'elle est définie au début du siècle. Ces traits sont déterminés par la «biologisation » d'un corps fait de sensibilité féminine et doté par la «nature » d'un sentiment maternel, occultant de fait sa contribution économique à la société.

Or, la consultation des données fournies par le recensement de la population libre dément cette vision. En 1819殹 ${ }^{4}$, sur une liste de 645 ménages de la région de Saint-Paul, on dénombrait 7 ménages dont le chef de famille se déclarait « couturière "; en $1848^{5}$, sur 1395 ménages recensés, $96^{6}$ femmes chef de famille soit près de $7 \%$ des ménages déclarent l'activité de couturière, ce qui représente $25 \%$ des 392 ménages dont le chef de famille est une femme et possédant moins de cinq esclaves. Ainsi donc, en dépit d'un discours dominant niant ou minorant l'exercice d'une activité professionnelle aussi bien masculine que féminine, le travail féminin, autre que celui de ménagère, est une réalité de la vie quotidienne bourbonnaise, ce que constate par exemple, le Docteur Yvan, de passage dans l'île en $1843^{7}$. L'augmentation de la mention " couturière » à la rubrique "profession" entre 1819 et 1848 alors que d'autres options, comme celle d'» habitante ${ }^{8}$ ou celle de "domestique » existent, ne peut pas être considérée comme fortuite. 
3 L'affirmation d'une identité professionnelle serait-elle la manifestation d'une dynamique sociale, ainsi que le suggère la définition du terme "profession »: " genre d'occupation que quelqu'un exerce notoirement", mais aussi "action de déclarer hautement, déclaration que fait quelqu'un de ses opinions " ${ }^{9}$. Compte tenu des sources utilisées, à savoir les recensements individuels des ménages, document officiel dont l'objectif est de cerner au mieux les réalités démographiques et économiques pour une gestion optimale du territoire, "faire profession de couturière ", c'est afficher une identité sociale qui sera enregistrée. Mais afficher sa profession, c'est aussi répondre aux injonctions de l'autorité publique en adhérant aux normes sociales qui ont toujours, dans la Colonie, une double origine.

Ce sont ces deux axes que nous développerons en esquissant le profil sociologique des couturières en fonction de trois critères : la composition de la famille, les patrimoines foncier et servile. Il s'agira de vérifier le poids des contraintes économiques dans le choix d'une activité professionnelle. Cette vision statique sera développée par une mise en situation sociale des individus à travers les représentations de l'activité qui sont tout à la fois des modèles normatifs et/ou idéalisés et des facteurs de la dynamique sociale. D'origine institutionnelle ou individuelle, les représentations sous-tendent les projets de société, explicites à Bourbon au cours du premier XIXe siècle. Mais ceux-ci doivent composer avec les représentations individuelles façonnées par des stratégies déterminées par les intérêts particuliers. Nous avons donc limité notre étude statistique à l'année 1847, c'est-à-dire à la veille de l'Abolition de l'esclavage, période cruciale pour le devenir de l'île qui a dû, dans la perspective de la disparition de l'esclavage, repenser la question du travail et son corollaire, la socialisation des agents économiques virtuellement libres de disposer de leur force de travail.

\section{Le profil sociologique des « couturières »}

5 Nous avons donc d'abord recensé les «couturières » et tenté d'établir leur profil sociologique, tel qu'il se dessine à travers les documents administratifs de l'année 1847, les opérations de recensement s'effectuant entre le $1^{\text {er }}$ janvier et le 15 février ${ }^{10}$. Ces couturières sont des chefs de famille monoparentale et majoritairement des mères célibataires comme l'indique la mention « demoiselle " précédant le patronyme. Seules deux d'entre elles sont veuves et une seule est mariée. L'étude des recensements permet de dégager un constat : la moitié de ces « couturières » ont en charge, ou ont eu à élever avant la date du recensement, plus d'un enfant. Pour établir une comparaison, nous avons sélectionné les "habitantes", chefs de famille monoparentale, propriétaires de moins de cinq esclaves, c'est-à-dire celles qui sont considérées comme indigentes ou en voie de paupérisation, en référence aux critères retenus par la Feuille Hebdomadaire de $1841{ }^{11}$ qui distingue les propriétaires de 10 esclaves "dans une position indépendante et assurée » et ceux qui ne possèdent aucun esclave et qui sont considérés comme "nécessiteux ». La comparaison met en évidence, pour l'année de référence, un pourcentage du nombre d'enfants légèrement supérieur chez les " couturières ». En l'état, cette dernière donnée n'est qu'indicative. La mention du père ou du compagnon apparaît en filigrane, à l'exemple de la « demoiselle Payet », dont les quatre enfants ont été reconnus par le "père Grimaud", ou de "Josèphe Grondin " vivant sous le toit de "Emerlin », dont l'enfant est déclaré vivant sous le toit de la mère, alors que celle-ci n'apparaît pas dans les feuilles en tant que déclarante. 
6 Nous avons ensuite tenté une approche économique en rapprochant les deux catégories précédentes. La différence socio-économique entre " habitante » et « couturière » est plus visible au niveau du patrimoine servile ou foncier comme l'indiquent les tableaux 1 et 2 .

\begin{tabular}{|c|c|c|c|c|c|c|c|c|}
\hline Nombre d'esclaves & 0 & 1 & 2 & 3 & 4 & 5 & $>5$ & Total \\
\hline Couturière & 82 & 10 & 2 & 0 & 2 & 0 & 0 & 96 \\
\hline$\%$ & 85,5 & 10,5 & 2,0 & 0,0 & 2,0 & 0,0 & 0,0 & 100,0 \\
\hline Habitante & 31 & 10 & 3 & 3 & 5 & 1 & 27 & 80 \\
\hline$\%$ & 38,7 & 12,5 & 3,7 & 3,7 & 6,3 & 1,3 & 33,8 & 100,0 \\
\hline
\end{tabular}

Tableau 1 : Distribution des ménages féminins selon le patrimoine servile

\begin{tabular}{|c|c|c|c|c|c|c|c|}
\hline $\begin{array}{c}\text { Patrimoine } \\
\text { en m }^{2}\end{array}$ & $\begin{array}{c}\text { Sans } \\
\text { patrimoine }\end{array}$ & $<500$ & $500-1499$ & $1500-3499$ & $? 3500$ & $\begin{array}{c}\text { patrimoine } \\
\text { imprécis }\end{array}$ & $\begin{array}{c}\text { Emplace- } \\
\text { ment bàti }\end{array}$ \\
\hline Couturière & 56 & 2 & 5 & 3 & 5 & 5 & 20 \\
\hline Habitante & 25 & 1 & 1 & 3 & 27 & 13 & 10 \\
\hline
\end{tabular}

Tableau 2 : Distribution des ménages féminins selon le patrimoine foncier

7 Ainsi, l'absence de patrimoine foncier ou servile, indice de pauvreté des ménages, expliquerait le choix de cette identité professionnelle. Toutefois, parmi celles qui se déclarent "habitantes ", 25 n'ont plus de terre et pour 13 autres cas, les propriétés semblent être des indivis ou des lanières allant du littoral au sommet des montagnes. Quelques "couturières" ont une propriété foncière comparable à celle de certaines " habitantes ». Ainsi, la "Demoiselle Cuvelier ", mère de 10 enfants, qui possède un esclave et détient une propriété à « Tête Dure au Ruisseau de $18 \mathrm{~m}$ de large et de 2400 $\mathrm{m}$ de profondeur ", se déclare " couturière ", tandis que la "demoiselle Gardenat ", quatre enfants, un esclave, sans propriété, se présente comme «habitante ». Nous pourrions multiplier les exemples car, a priori, l'explication du choix de la profession de couturière par le niveau de richesse ne saurait rendre compte de la situation. En effet, l'absence de patrimoine ne conduit pas toutes les femmes à embrasser une activité professionnelle. De plus, d'autres activités assurent la subsistance des pauvres de l'île et en particulier la vannerie dont le savoir-faire est ancien. Mais un dernier point a retenu notre attention: dans la partie réservée aux individus vivant sous le même toit, on trouve à plusieurs reprises l'indication de filles exerçant cette activité que ce soit chez les « habitantes" ou chez les « couturières ». L'activité de couturière semble être une affaire de famille et se présente comme un métier d'avenir particulièrement prisé puisqu'il est embrassé par au moins deux générations.

\section{La « couturière » dans son réseau social}

Pour mieux cerner l'activité des couturières, nous avons eu recours au témoignage du planteur Renoyal de Lescouble et aux témoignages littéraires, seules sources disponibles permettant de replacer la couturière dans son réseau social. 
9 En France, la naissance de la corporation des couturières date de 1675 et constitue, selon Daniel Roche ${ }^{12}$, une étape de la conquête du secteur de l'habillement par les femmes; à Bourbon, l'activité semble associée au travail servile. Rappelons que les ateliers du Roi comptaient 14 couturières. Toutes les plantations ne possédaient pas de couturière, comme l'indique le Journal de Lescouble, et le recours aux services extérieurs semble avoir été la règle, malgré la contribution des femmes créoles dont témoigne Auguste Billard ${ }^{13}$. À plusieurs reprises, Lescouble fait intervenir sa fille ou des dames de son entourage pour lui « faire faire » des chemises et des pantalons ainsi qu'il le note dans son Journal le vendredi 11 avril $1828{ }^{14}$. Ces sources nous renseignent partiellement sur le statut social des « couturières ». La seule précision fournie par le Journal de Lescouble concerne Euranie, l'esclave qu'il affranchit en juillet 1824 et qui se place sous sa protection. Le dimanche 28 novembre 1824, il note que : « la ménagère de Pinard est venue voir Euranie et l'a priée de lui tailler une robe ${ }^{15}$.

10 Le Docteur Yvan, en visite dans l'île en 1843, apporte d'autres précisions; dans une demeure de riches Créoles, il décrit la scène suivante : «La maîtresse de maison lisait à demi couchée sur un divan. Dans un coin, à distance, se tenaient trois ou quatre femmes de diverses nuances; elles cousaient en babillant à demi-voix " ${ }^{16}$. Cette scène confirme la représentation classique de la femme créole et souligne la répartition des tâches dans une maison de la bonne société de l'île. La couture, et non pas tous les travaux d'aiguille, est une activité réservée aux domestiques. Cela est corroboré par des extraits du Bourbon Pittoresque d'Eugène Dayot ${ }^{17}$. Dans le système servile, cette activité est donc réservée aux domestiques dont le sort est plus enviable que celui de l'esclave de pioche. Mais le récit d'Yvan révèle par ailleurs une dynamique interne au système servile. En visitant un camp d'esclaves, il note une différence notable entre les "cases». Le premier type est comparé à un chenil, mais dans la deuxième «maisonnette » qui se rapproche du "foyer modèle » européen, le visiteur aperçoit "un coffre en bois destiné à renfermer des vêtements ». "Cette cabane ", poursuit l'observateur,

[...] était habitée par une jeune négresse, laquelle, lorsque nous entrâmes, cousait silencieusement assise devant la fenêtre, chose rare assurément dans une case à nègres. La jeune esclave portait un jupon bleu de toile de coton, et un grand fichu blanc était modestement croisé devant sa poitrine. ${ }^{18}$

11 Le propos d'Yvan est révélateur de la diffusion de certaines normes sociales métropolitaines. Ces dernières sont véhiculées dans l'île par les institutions religieuses et/ou scolaires et sont celles de la «bonne société » créole. La description du voyageur insiste sur les signes visibles de l'ascension sociale comme l'ameublement, l'ordre et la chaleur de la maison et surtout - d'où la surprise de l'observateur - l'occupation de l'esclave qui, en quelque sorte, lui restitue, à ses yeux, une humanité. Cette tâche rapproche en effet l'esclave de la maitresse en lui offrant le luxe de s'occuper de son foyer ${ }^{19}$. Dans le système esclavagiste, être couturière semble constituer une promotion sociale puisqu'elle ramène la femme à l'intérieur de la maison, la libérant de la condition servile dont l'image reste liée aux champs. Le sort d'Euranie, affranchie et maitresse de Lescouble, semble confirmer que l'activité de couturière est la marque de la liberté et expliquerait l'adoption de cette activité par les Libres. D'une manière générale, l'engouement pour cette profession de la part de toutes les catégories, en particulier des femmes libres recensées plus haut, tient sans doute à la fonction repoussoir des travaux des champs, le travail agricole étant assimilé à l'esclavage ; 
l'absence de conjoint associée à celle du patrimoine servile, au regard des constats de la première partie, expliquerait aussi l'adoption de cette identité professionnelle.

\section{Un effet de la moralisation du « prolétariat colonial »?}

12 Cependant, compte tenu de la possibilité d'opter pour la mention "sans profession ", option choisie par quelques femmes, l'explication du choix doit aussi prendre en compte le contexte général qui est celui de la valorisation du travail - qui intervient en France, selon D. Méda, dans le premier tiers du XIXe siècle - et qui fait partie du dispositif de socialisation du prolétariat préconisé par les penseurs sociaux en charge de l'économie sociale ${ }^{20}$. La valeur intégratrice dévolue au travail, selon les économistes, impose à chaque individu une identification sociale autre que patronymique. L'acceptation de cette norme doit être considérée comme un indice du procès de « moralisation » des classes pauvres engagé à Bourbon avant 1848.

Il faut rappeler le contexte général de l'île qui doit gérer une importante mutation économique. Pour affronter la concurrence des betteraviers, les sucriers envisagent l'utilisation d'une main d'œuvre libre pléthorique mais réputée paresseuse, vagabonde et hostile au travail ${ }^{21}$. Or, les tentatives de formation, rapportées par Raoul Lucas dans Bourbon à l'école (1997), se sont avérées infructueuses. À la veille de ce qui est pressenti comme un bouleversement majeur de la société créole, la préoccupation de moraliser les classes indigentes par le biais des enfants et des femmes est une constante dans la presse. Mais les arguments en faveur du «travail » de la femme ne sont pas nombreux puisque celle-ci, en fait, n'est pas la principale cible à atteindre, comme le montrent les exemples qui suivent.

Les deux propositions suivantes ont en commun l'ambition de régler la question sociale. La première, celle de Richard, date de 1821, période de conversion de l'île à l'agro-industrie et de la prise de conscience de la question du " paupérisme colonial » et des moyens de son extinction :

Quant aux jeunes filles on pourrait [les] employer à mille fonctions domestiques,

[...], fileuses, couseuses, repasseuses, lavandières et toutes les occupations qui n'ont rien de servile ni d'avilissant, gardant les esclaves pour cette dernière sorte de fonctions ${ }^{22}$.

Pour Richard, l'emploi des femmes s'appuie sur le concept d'utilité sociale mais dans le respect de la hiérarchie imposée par la structure esclavagiste dont l'abolition n'était pas encore à l'ordre du jour à cette date. Ces deux aspects respectent aussi la répartition sexuée des rôles plus conforme aux nouvelles valeurs de la politique économique. Sa proposition s'inscrit dans le cadre de l'éradication du paupérisme par la mise au travail généralisée des indigents mais vise principalement la population masculine.

La seconde, celle de Vassal, correspondant occasionnel des hebdomadaires bourbonnais, propose en 1844 d'encourager le développement de la sériciculture pour "donner du pain » et pour «occuper les malheureux» car, précise-t-il : «[...] cette industrie, [...] si facile, est pour ainsi dire l'industrie spéciale des femmes. Celles-ci, étant occupées, sauront bien se faire seconder par les hommes, qui ne croupiront plus dans la paresse dont on se plaint $[. ..]{ }^{\text {» }}{ }^{23}$. Il semble exprimer à voix haute la préoccupation centrale de l'époque qui est celle de la mise à la disposition de l'industrie sucrière d'une main d'œuvre jusqu'alors flottante et donc inutile, voire dangereuse. Le 
travail de la femme doit servir d'instrument de socialisation: il s'agit de régler la question sociale en contrôlant la famille dont la femme est le pivot; c'est grâce à elle que se résoudront les problèmes de vagabondage et l'absence d'intérêt pour le travail de la population libre et incontrôlable. Cette proposition rejoint celle des philanthropes ${ }^{24}$ engagés dans une politique de résorption du paupérisme qui, constatant l'impossibilité de modifier les règles de la nouvelle économie, préconisent de modifier le comportement des pauvres face au travail en leur inculquant les nouvelles valeurs. La mesure proposée par Vassal atteindrait ainsi un double objectif : la mise au travail des hommes et la possibilité pour les autorités de recenser les populations en état de travailler, objectif principal à atteindre comme le rappelle le rapport du Comité d'agriculture réuni en 1841 et repris dans la Feuille Hebdomadaire ${ }^{25}$. Dans ce rapport, les membres du Comité d'agriculture déplorent l'impossibilité légale de mener à bien les poursuites contre "les individus «sans aveu » c'est-à-dire en état de travailler, n'ayant ni moyens de subsistance, ni métier, mais qui «[...] remplissent une condition de manière légale ; c'est celle du domicile certain [...] ». Le Comité propose d'introduire le livret pour les "ouvriers cultivateurs" et pour tous les individus "[...] qui ne peuvent exister sans un travail personnel » et, lors du recensement annuel, de classer les individus qui ne possèdent pas de moyens d'existence dans la catégorie des "gens de travail ». Mais ces dernières propositions étaient-elles connues du grand public et visaient- elles la population féminine? Dans l'affirmative, la multiplication des "couturières" exprimerait ainsi une volonté de se mettre aux normes de manière préventive.

17 Cette dernière mesure, appliquée seulement en 1848, témoigne en faveur d'une réflexion sur le paupérisme et complète le dispositif de moralisation par le travail déjà mise en œuvre par des institutions de bienfaisance qui accueillaient les jeunes filles. Il s'agit de faire de la femme une intermédiaire chargée de transmettre des valeurs croit-on - étrangères à l'île. Ce dessein est identique à celui de l'école dont l'enseignement vise à encadrer les esprits pour maintenir l'ordre social structuré par le respect des sphères et des rôles sociaux ainsi que le rappelle l'extrait de ce rapport lu lors de la distribution des prix au Collège Royal de septembre 1837. Les délégués de la commission d'instruction se sont en effet particulièrement félicités de ce que « [...] dans les écoles de jeunes filles [...] l'instruction littéraire ne faisait pas négliger l'apprentissage et la pratique de connaissances plus modestes, mais dont l'application deviendra plus tard une nécessité domestique [...]» ${ }^{26}$. L'ambition d'» habituer » les jeunes filles « aux ouvrages de leur sexe ${ }^{27}$ est présente dans toutes les écoles de filles, conformément aux normes de l'époque; les jeunes filles fréquentant l'établissement des Dames de Charité ne reçoivent qu'un enseignement scientifique rudimentaire.

Nous n'avons pas pu encore établir que nos « couturières » ont suivi cet enseignement, mais nous pouvons supposer que certaines d'entre elles ont été scolarisées ou ont été en contact avec des enfants scolarisés. Au-delà des cas individuels, l'influence de l'école sur la population bourbonnaise est une hypothèse vraisemblable au regard des chiffres proposés par le rapport Greslan de 1837 : le nombre des écoles autorisées, y compris le Collège Royal, est de 46, soit 28 pour les garçons et 18 pour les jeunes filles, pour 2043 enfants scolarisés. Ici, comme en Europe, la formation limitée des femmes s'appuie sur le principe de la faiblesse du sexe et de l'existence d'aptitudes dites « naturelles » qui la vouent à la fonction maternelle et domestique. On y voit la manifestation de la séparation des sphères, héritage des Lumières mais qui se confirme au début du XIXe siècle, la sphère privée étant centrée sur la maison et la famille, gestionnaire des 
intérêts privés ${ }^{28}$, soustraite à l'autorité des pouvoirs. La cellule familiale se trouve investie de nouvelles attributions : la reproduction biologique et celle des valeurs. La famille devient le lieu de la première socialisation.

Cette préoccupation est partagée par les insulaires. Selon la Feuille Hebdomadaire du 13 février 1839, la famille est le noyau autour duquel gravitent les problèmes de société ; il s'agit tout à la fois d'extraire les enfants d'un milieu familial dont l'influence est jugée nocive et de créer des conditions nouvelles pour la mise en place d'une nouvelle société dont la cellule de base serait la famille. L'activité féminine ne peut être envisagée que si elle est compatible avec ce rôle social. Il s'agit dès lors d'inclure les femmes dans un réseau d'échanges économiques, mais, dans cette nouvelle configuration, la maison reste le point d'ancrage.

20 Si pour la femme pauvre, le travail de couturière à la maison, qui occupe le corps et l'esprit, est une nécessité afin de l'éloigner du vice de la prostitution, ce travail ne saurait être que "féminin », suivant l'acception qui prévaut à cette époque, c'est-à-dire qu'il prolonge les fonctions "naturelles" de la femme, à savoir protéger (fonction principale du vêtement), aider (la confection de vêtement ne crée pas de richesse macro-économique mais a une fonction sociale), et " parer » (ce qui classe cette activité dans les services). Mais en éduquant au travail la femme au point que cela devienne un " sentiment inné », pour reprendre une expression de la Feuille Hebdomadaire ${ }^{29}$, l'auteur de l'article a, semble-t-il, assimilé le rôle biologique de transmission de la vie à celui de la transmission des valeurs. En effet, le thème récurrent sollicité pour expliquer la paresse de la population libre, et a fortiori esclave, est le thème biologique; la population libre est biologiquement conditionnée pour l'oisiveté et ce sont des valeurs extérieures à l'île qui pourraient régénérer l'espèce; c'est une autre variante de la proposition faite par Thomas en 1828 pour introduire le goût du travail ${ }^{30}$. Toutefois, l'idéologie sans coercition ne peut avoir un effet mécanique. Dans le choix de la profession de couturière entrent en ligne de compte des considérations de statut, la nécessité plus ou moins consciente d'adopter la norme, ou en tout cas, l'illusion d'y adhérer, mais, nous l'avons dit à plusieurs reprises, il existe d'autres options. Pour reprendre l'analyse de Bernard Lepetit, il n'y a pas de réception passive de l'idéologie ${ }^{31}$. Ceci nous amène à réfléchir sur un autre aspect de la couture, à savoir la production.

\section{La portée symbolique de la production vestimentaire.}

21 Le nombre relativement important de couturières relève peut-être de l'affichage, mais suppose aussi une demande suffisante pour le justifier officiellement. Cette demande répond certes à des nécessités primaires, mais, dans le contexte insulaire, l'investissement symbolique de la parure et du vêtement ne peut être ignoré. La question de l'apparence vestimentaire est souvent mise en avant dans les récits de voyage, expression d'un savoir préconstruit, fruit de lectures antérieures qui aboutirait à la description stéréotypée de la réalité sociale, mais pourtant des témoignages locaux vont dans le même sens. Ainsi, le planteur Lescouble mentionne dans son journal ses achats de tissus, ses commandes de vêtements, qui sont relativement importantes, comme il le fait pour ses travaux agricoles. Il s'agit pour une part de satisfaire aux exigences légales concernant l'habillement des esclaves, mais ses commandes portent aussi sur l'achat de tissus pour lui et sa femme. C'est que l'élégance des Créoles participe du stéréotype des femmes bourbonnaises quelle que soit leur condition 
sociale ${ }^{32}$, le stéréotype étant la représentation caricaturale d'un trait culturel. Toutefois, la question de l'apparence vestimentaire a retenu l'attention d'historiens comme Daniel Roche qui, dans son ouvrage La culture des apparences de la fin du XVIIIe siècle au XIXe siècle, a étudié ces indices matériels comme des témoignages sociologiques. C'est dans cette optique que nous tenterons de lire la réalité sociale de l'île au début du XIXe siècle.

Daniel Roche montre en effet que l'élégance vestimentaire féminine devient un indice de la sexualisation de la vie sociale. Mais l'apparence vestimentaire est aussi investie d'une fonction d'identification sociale valable en tous lieux ${ }^{33}$. Dans la société bourbonnaise du premier XIXe siècle, le système servile génère une dynamique qui traverse une société possédant les caractères d'un "ancien régime vestimentaire ", régime marqué par « [...] l'inertie et l'immobilité [...]; la coïncidence entre costume et position sociale ; la volonté de contrôle, qui dicte aux autorités les lois somptuaires et à tous normes de l'étiquette et conformisme des usages ». ${ }^{34}$

Nous sommes dans une société qui a dû procéder à la codification des apparences vestimentaires par l'ordonnance du 18 mai 1819 qui avait rappelé les normes. La codification de l'apparence vestimentaire entendait permettre de faire respecter la hiérarchie sociale car elle exigeait de paraitre selon sa condition et offrait par là une grille de lecture permettant la visualisation et le décodage des différences sociales. Cette fonction d'identification est d'ailleurs fréquemment soulignée par les voyageurs dans leurs récits. L'attribution d'un " costume » assignait ainsi une identité sociale et assurait la supériorité de "l'état» sur l'individu. Le texte qui accompagne la lithographie d'Antoine Roussin du 14 octobre 1848 intitulé "Conversation politique » 35 confirme la pérennité de cet " ancien régime vestimentaire ».

24 Mais, la nécessité même de prendre une ordonnance en 1819, ordonnance qui s'apparente à une loi somptuaire, révèle déjà la transgression de la codification des apparences, facteur de désordre social. Les esclaves ont l'autorisation de porter un mouchoir de tête à condition que le tissu soit de qualité commune. Il leur est interdit de porter «des mousselines, dentelles, gazes, soieries, rubans, châles de laine et de mérinos ", « objet de luxe au-dessus de l'état de l'esclavage». Tout ceci révèle une inadéquation entre la norme et la réalité sociale : la transgression des "états » vérifiée par les témoignages des contemporains et dont, par exemple, le récit d'Yvan rend compte :

Les jeunes négresses ainsi honorées des attentions passionnées de leurs maîtres ne sont pas assujetties aux rudes labeurs des champs; elles sont employées aux travaux d'intérieur; souvent même elles ne quittent pas leur case. Leur mine contraste singulièrement avec les misérables haillons de leurs compagnes, et ne manque pas d'une certaine élégance; les étoffes dont elles se parent empruntent leur éclat à toutes les couleurs de l'arc-en-ciel. ${ }^{36}$

Cette transgression des usages révèle des tiraillements à l'intérieur d'une société où la mobilité sociale semble pourtant réduite ; le paraître vient visualiser et attester de la possibilité de changer d'état. L'apparence vestimentaire est aussi une manière de marquer son statut et de se différencier des esclaves. En effet, Sudel Fuma ${ }^{37}$ rappelle que malgré l'obligation officielle de fournir aux esclaves des habits, obligation réitérée en juin 1846, la distribution de vêtements est restée parcimonieuse, en particulier sur les petites plantations. De plus, il signale qu'aux champs, les esclaves conservaient des vêtements très légers mais n'hésitaient pas à s'offrir des vêtements de qualité supérieure à ceux qui leur étaient proposés. 

une profession exercée jusque-là par des esclaves, conduit à l'égalisation des conditions que le système condamne et tente d'éviter en encourageant la compétition entre les groupes sociaux. Or les pratiques, telles qu'elles se dessinent à travers les déclarations individuelles, rendent caducs les efforts de ceux qui souhaitent maintenir la hiérarchie sociale en respectant les stratifications professionnelles. Rappelons qu'au recensement de 1819, six "couturières » sur sept étaient des «Libres» ou des "Affranchies"; seule une certaine "Veuve Jouan" n'était pas recensée dans cette catégorie. En 1848, apparaissent dans le recensement pour quatre d'entre elles la mention, «malgache » ou " née à Madagascar». Les patronymes, par ailleurs, laissent aussi supposer un nombre assez important de descendants de Libres ou d'Affranchies que, faute de temps, nous n'avons cependant pas pu vérifier. En vingt-neuf années, la profession exercée jusque-là par des descendants d'esclaves est embrassée par des femmes qui n'appartiennent pas à cette catégorie et qui l'exercent aux côtés de femmes qui ne sont pas blanches.

En deuxième lieu, l'activité de couturière est liée au système servile, mais s'est transformée en un outil de promotion et d'émancipation. La question qui se pose est la suivante: est-ce un signe d'appropriation par ces dernières d'un système de valeur exogènes, véhiculées par les institutions d'enseignement, ou une «créolisation « des valeurs? En effet, l'activité semble avoir perdu sa marque servile puisque des libres de naissance l'exercent pour satisfaire une demande qui aspire à effacer les traces de la servilité. 


\section{BIBLIOGRAPHIE}

Archives

Archives Départementales de la Réunion (ADR) 2Fi 1992, panneau 343 de l'exposition.

ADR 57 M 1 : Mémoire de Richard « Moyens proposés afin d'utiliser la population inactive de l'île Bourbon ", 1821.

Feuilles de Recensements de Saint-Paul, 6 M 584-588, ADR.

Feuilles de Recensements de Saint-Paul, 6 M 719-724, ADR.

Feuille Hebdomadaire de l'Ile Bourbon (FHIB) n 1050 du 13 février 1839, ADR.

FHIB nº 1050 du 13 février 1839, ADR.

FHIB n 1156 du 24 février 1841, ADR : « Questions relatives à la diminution des bras ainsi que celles qui se rattachent à l'état du paupérisme colonial. Nécessité de prendre des mesures contre le paupérisme colonial, préalable à la modification du régime de l'esclavage ».

FHIB n 1314 du 6 mars 1844, ADR.

FHIB n 975 du 6 septembre 1837, ADR : Discours de M. de Greslan chargé de rendre compte des opérations de la Commission d'Instruction Publique pour l'arrondissement au vent pendant l'année écoulée.

FHIB, $n^{\circ} 1156$ du 24 février 1841, ADR.

Indicateur colonial, $\mathrm{n}^{\circ} 491$ du 24 août 1844, ADR.

Ouvrages cités

ADR, 1998, Regards croisés sur l'esclavage 1794-1848, Saint-Denis, CNH la Réunion \& Somogy \& Éditions d'Art.

AZÉMA Georges, 1859, Histoire de l'île Bourbon depuis 1643 jusqu'au 20 décembre 1848, Paris, Plon.

BILLIARD Auguste, 19902, Voyage aux colonies orientales, Lettres écrites à M. le Comte de Montalivet, ancien ministre de l'Intérieur pendant les années 1817 à 1820, Paris, Ladvocat, 18221, 490 p. ; réédition Saint-Denis, Collection « Mascarin ».

DAYOT Eugène, 1977, Bourbon pittoresque [1848], dans Euvres choisies, édition annotée par Jacques Lougnon, Saint-Denis, Imprimerie Dionysienne.

DELABARRE de NANTEUIL Comte A. de, 1843-1844, Législation de l'île Bourbon, Paris, Gros, 3 vol.

FUMA Sudel, 1979, Esclaves et citoyens, le destin de 62000 Réunionnais. Histoire de l'insertion des affranchis de 1848 dans la société réunionnaise, Saint-Denis, Fondation pour la recherche et le développement dans l'océan Indien.

_, 1992, L'esclavagisme à la Réunion, 1794-1848, Paris, L'Harmattan/Université de la Réunion. GRONDIN Reine-Claude, 1998, La construction historique des catégories sociales. L'exemple des « Petits Blancs des hauts » à l'île de La Réunion, Mémoire de DEA, Université de la Réunion.

HATZFELD Adolphe, DARMESTETER Arsène, THOMAS Antoine, 1964, Dictionnaire Général de la langue française du commencement du XVIIe siècle jusqu'à nos jours, Paris, Delagrave. 
LEPETIT Bernard, 1999, Carnet de croquis, Paris, Albin Michel.

LESCOUBLE Jean-Baptiste Renoyal de, 1990, Journal d'un colon de l'île Bourbon, 3 vol. Paris, L'Harmattan/Éditions du Tramail.

LUCAS Raoul, 1997, Bourbon à l'école, 1815-1946, Saint-André, Océan éditions.

MÉDA Dominique, 1995, Le travail, une valeur en voie de disparition, Paris, Aubier.

PELLEGRIN Nicole, 1999, « Les vertus de 'l'ouvrage'. La féminisation des travaux d'aiguille, XVIeXVIIe siècle ", Revue d'histoire moderne et contemporaine, $\mathrm{n}^{\circ}$ 46-4, oct.-déc., p. 747-769.

PERROT Michèle, 1998, Les Femmes ou les silences de l'histoire, Paris, Flammarion.

PROCACCI Giovanna, 1993, Gouverner la misère : la question sociale en France (1789-1848), Paris, Éd. du Seuil.

ROCHE Daniel, 1989, La culture des apparences. Une histoire du vêtement XVIIe-XVIIIe siècle, Paris, Fayard.

THOMAS, 1828, Essai de statistique de l'île Bourbon considérée dans sa topographie, sa population, son agriculture, son commerce, Paris, Bachelier et Selligne, 2 vol.

VERDIER Yvonne, 1997, Façons de dire, Façons de Faire, Paris, Gallimard.

YVAN Docteur M., 1855, De France en Chine, Paris, Hachette.

\section{NOTES}

1. Verdier 1997.

2. Ile Bourbon jusqu'en 1848.

3. Azéma 1859 : 139.

4. Feuilles de Recensements de Saint-Paul, 6 M 584-588, Archives Départementales de la Réunion (ADR).

5. Feuilles de Recensements de Saint-Paul, 6 M 719-724, ADR.

6. Pour un total de 116 professions féminines autres qu'» habitante» - terme désignant la profession de planteur - à l'origine propriétaire d'une "habitation». Les autres professions féminines se répartissent de la manière suivante: 2 bazardières (marchande de légumes ambulante), 5 marchandes, 2 repasseuses, 1 domestique, 1 sage-femme, 3 laveuses, 1 servante, 3 brodeuses, 1 débitante et une " échappière ».

7. Yvan $1855: 176$.

8. Synonyme de « cultivatrice », cf. note 6 .

9. Hatzfeld, Darmesteter, Thomas 1964.

10. Delabarre de Nanteuil 1843-1844.

11. ADR, Feuille Hebdomadaire de l'Ile Bourbon (FHIB), nº 1156 du 24 février 1841.

12. Roche 1989.

13. Billiard 1822 : 237-238.

14. Lescouble 1990: 664. La légende de la caricature figurant dans le catalogue d'exposition «Regards croisés sur l'esclavage 1794-1848 », qui présente les conséquences de l'Abolition dans l'île, confirme ce fait ; ADR 1998.

15. Lescouble $1990: 347$.

16. Yvan 1855 : 151

17. Dayot $1848: 59$.

18. Yvan $1855: 163$. 
19. Billiard $1822: 237-238$.

20. Meda 1995 ; Procacci 1993.

21. Sur ce point, voir Grondin 1998.

22. ADR 57 M 1 : Mémoire de Richard « Moyens proposés afin d'utiliser la population inactive de l'île Bourbon », 1821. Richard est un propriétaire de Sainte-Suzanne.

23. Indicateur colonial, $\mathrm{n}^{\circ} 491 \mathrm{du} 24$ août 1844, ADR.

24. Voir Procacci 1993.

25. FHIB n 1156 du 24 février 1841, ADR : «Questions relatives à la diminution des bras ainsi que celles qui se rattachent à l'état du paupérisme colonial. Nécessité de prendre des mesures contre le paupérisme colonial, préalable à la modification du régime de l'esclavage ».

26. FHIB n 975 du 6 septembre 1837, ADR : Discours de M. de Greslan chargé de rendre compte des opérations de la Commission d'Instruction Publique pour l'arrondissement au vent pendant l'année écoulée.

27. FHIB n 1050 du 13 février 1839, ADR.

28. Voir sur ce point Perrot 1998.

29. FHIB $\mathrm{n}^{\circ} 1050$ du 13 février 1839, ADR.

30. Thomas, $1828: 287$.

31. Lepetit $1999: 150$.

32. Yvan $1855: 155,182,176$.

33. Roche $1989: 50$.

34. Roche $1989: 60$.

35. Antoine Roussin, Album de la Réunion, cité par ADR, 1998 : 225. « [...] n'a pas Gouvernère ça, nana zabit com' Mesié Alidor [...] ça Gouvernère pour noirs!» traduction: «Ce n'est pas un gouverneur, celui-là. Il est habillé comme monsieur Alidor [...] C'est un gouverneur pour les Noirs! ».

36. Yvan $1855: 182$.

37. Fuma $1992: 86$

38. La tendance se confirme après l'Abolition. Sudel Fuma (1979) a recensé en 184917 couturières sur un échantillon de 310 affranchis (ADR, 4E6 299).

\section{RÉSUMÉS}

La couturière à la Réunion: figure ou actrice sociale? Cet article aborde les stratégies professionnelles des femmes libres dans une société esclavagiste, Bourbon (La Réunion) en 1847, confrontée à la nécessité d'introduire le travail libre. Dans ce contexte, les femme sont perçues comme actrices du changement social. L'augmentation des déclarations de l'activité de couturière révèle aussi l'appropriation de nouvelles valeurs, soumises cependant aux contraintes du genre et de l'environnement servile.

The seamstress in the Reunion island: figure or social actress? This article analyses the professional strategies of free women in a slave society - Bourbon (the Reunion) - in 1847 which was forced to introduce free work. In this context, women were perceived as actresses in social change. The increasing number of statements about the activity of seamstresses reveals the appropriation of new values, conditioned however by the gender constraints of an unfree society. 
INDEX

Index géographique : île de la Réunion

Mots-clés : esclavage, hommes libres, moralisation, genre, travail libre

Keywords : clothing regime, free persons, free work, moralization, Reunion island, slavery, gender

\section{AUTEUR}

\section{REINE-CLAUDE GRONDIN}

Reine-Claude GRONDIN est doctorante à Paris I. Elle a collaboré à l'ouvrage dirigé par Claude Lauzun, La colonisation : droit d'inventaire. Les isles : les Antilles et la Réunion, (Paris, A. Colin, 2004), et au Dictionnaire de la Colonisation dirigé par Claude Liauzu, (Paris, Larousse, 2007). 